\title{
ENERGY DECAY FOR DAMPED WAVE EQUATIONS ON PARTIALLY RECTANGULAR DOMAINS
}

\author{
Nicolas Burq ANd Michael Hitrik
}

\begin{abstract}
We consider the wave equation with a damping term on a partially rectangular planar domain, assuming that the damping is concentrated close to the nonrectangular part of the domain. Polynomial decay estimates for the energy of the solution are established.
\end{abstract}

\section{Introduction and statement of result}

The purpose of this note is to show how the methods of [4], [5] apply to estimate the energy decay rates for the damped wave equation on a class of planar domains, including some ergodic billiards. In a situation when the geometric control condition of Bardos, Lebeau, and Rauch [1] does not hold, we obtain a polynomial decay estimate for the energy of the damped wave, with respect to a stronger norm of the initial data. In order to formulate the main result, we shall begin by recalling some standard notation and assumptions.

Let $\Omega \subset \mathbf{R}^{n}, n \geq 2$, be a bounded connected domain, with $\partial \Omega \in C^{\infty}$. When $0 \leq a \in L^{\infty}(\Omega)$ is a non-negative smooth function on $\Omega$, we consider the following initial-boundary value problem,

$$
\left\{\begin{array}{l}
\left(-D_{t}^{2}-\Delta+2 i a(x) D_{t}\right) u=0, \quad(t, x) \in \mathbf{R}_{+} \times \Omega \\
u=0 \text { on } \mathbf{R}_{+} \times \partial \Omega, \\
\left.u\right|_{t=0}=u_{0} \in H_{0}^{1}(\Omega),\left.\quad D_{t} u\right|_{t=0}=u_{1} \in L^{2}(\Omega) .
\end{array}\right.
$$

Here $D_{t}=\partial_{t} / i$ and we shall assume throughout that the damping coefficient $a$ does not vanish identically.

Associated with the evolution problem (1.1) is the solution operator $U(t)=e^{i t A}$, $t \geq 0$, acting in the Hilbert space of the Cauchy data $H=H_{0}^{1}(\Omega) \times L^{2}(\Omega)$ and mapping $\left(u_{0}, u_{1}\right) \in H$ to $\left(u(t, \cdot), D_{t} u(t, \cdot)\right)$. Here we equip $H$ with the norm

$$
\left\|\left(\begin{array}{c}
u_{0} \\
u_{1}
\end{array}\right)\right\|_{H}^{2}=\left\|\nabla u_{0}\right\|_{L^{2}}^{2}+\left\|u_{1}\right\|_{L^{2}}^{2} .
$$

The infinitesimal generator $A$ of the semigroup $U(t)$ is the operator

$$
A=\left(\begin{array}{cc}
0 & 1 \\
-\Delta & 2 i a(x)
\end{array}\right): H \rightarrow H
$$

Received by the editors January 12, 2006.

2000 Mathematics Subject Classification. 35L05, 47A10, 47B44, 47D06, 49J20.

Key words and phrases. Damped wave equation, non-selfadjoint, partially rectangular, energy decay, geometric control, resolvent. 
with the domain $D(A)=\left(H^{2} \cap H_{0}^{1}\right) \times H_{0}^{1}$. It follows that the spectrum of $A$ is discrete, and from [8] we recall that if $\lambda \in \mathbf{C}$ is an eigenvalue of $A$ then $0<\operatorname{Im} \lambda \leq$ $2\|a\|_{L^{\infty}}$.

For future reference, let us notice that when $U \in D(A)$, then $\operatorname{Im}\langle A U, U\rangle_{H} \geq 0$ and hence

$$
\left\|(\lambda-A)^{-1}\right\|_{L(H, H)} \leq \frac{1}{|\operatorname{Im} \lambda|}, \quad \operatorname{Im} \lambda<0 .
$$

In this note we shall be concerned with the energy of the solution $u(x, t)$ of $(1.1)$ at time $t$,

$$
E(u, t)=\frac{1}{2} \int_{\Omega}\left(\left|\nabla_{x} u\right|^{2}+\left|D_{t} u\right|^{2}\right) d x .
$$

It is easily seen that $E(u, t)$ is nonincreasing as $t \rightarrow \infty$ and from [8] we may also recall that $E(u, t) \rightarrow 0$ as $t \rightarrow \infty$, for each $\left(u_{0}, u_{1}\right) \in H$. Under the geometric control condition stating that there exists $T_{0}>0$ such any billiard trajectory in $\Omega$ of length $\geq T_{0}$ meets the open set $\{x ; a(x)>0\}$, the uniform exponential decay of the energy has been established by Bardos, Lebeau, and Rauch [1]. In the general case, without any assumptions on the underlying dynamics, it has been proved by Lebeau [8] that the decay rate of the energy is always logarithmic, provided that the initial data in (1.1) are measured with respect to a stronger norm. In this note, we shall derive a polynomial decay estimate for the energy, for a class of planar domains and damping regions, in the case when the geometric control condition of [1] fails to hold.

We shall consider the class of partially rectangular domains $\Omega \subset \mathbf{R}^{2}$. By this we mean that $\Omega$ is connected, has a boundary that is piecewise $C^{\infty}$, and contains a rectangle $R \subset \Omega$, such that if we decompose the boundary of $R$ into pairs of parallel segments, $\partial R=\Gamma_{1} \cup \Gamma_{2}$, then $\Gamma_{j} \subset \partial \Omega$ for at least one $j$, say for $j=1$. We shall write $\Omega=R \cup W$, where $W$ is the non-rectangular part of $\Omega$.

Example. The Bunimovich stadium $S$, defined as the union of a rectangle $R=$ $\{(x, y) ; x \in[0,1], y \in[0,2 \beta]\}, \beta>0$, with two semicircular regions centered at $(0, \beta)$ and $(1, \beta)$, with radius $\beta$, which lie outside of $R$, is a partially rectangular domain. It is well known [2] that the geodesic flow in $S$, obeying the law of reflection at the boundary, is ergodic. In what follows, the ergodicity of the underlying classical flow in $\Omega$ will not play any special role in our considerations.

Theorem 1.1. Let $\Omega \subset \mathbf{R}^{2}$ be a partially rectangular domain, $\Omega=R \cup W$, and let $0 \leq a \in C(\bar{\Omega})$ be such that $a>0$ in $\bar{W}$, the closure of $W$ in $\mathbf{R}^{n}$. Then for each $k>0$ there exists a constant $C_{k}>0$ such that for each $\left(u_{0}, u_{1}\right) \in D\left(A^{k}\right)$ we have

$$
E(u, t)^{1 / 2} \leq C_{k} \frac{(\log t)^{\frac{k}{2}+1}}{t^{\frac{k}{2}}}\left\|\left(u_{0}, u_{1}\right)\right\|_{D\left(A^{k}\right)}, \quad t \geq 2 .
$$

Remark. We notice that in the case when the damping coefficient $a$ vanishes outside of a neighborhood of $\bar{W}$ in $\Omega$, the geometric control condition of [1] fails, due to the existence of the invariant set for the classical flow constituted by the bouncing ball orbits parallel to the pair of segments $\Gamma_{2}$ and staying within the rectangle $R$. 
Therefore (see [1]), no uniform decay estimate for the energy with respect to the energy norm of the initial data is possible.

Theorem 1.1 is proved in section 2. We shall finish this section by briefly discussing the issue of optimality of the bound (1.5). First, arguing heuristically on the classical level, let us notice that every ray starting in the interior of the rectangle and forming an angle of size $\frac{1}{k}, k \gg 1$, with the direction of $\Gamma_{2}$ will reach the non-rectangular part of the domain after a time which is of the order $\sim k$. When discussing the quantum picture, for simplicity, we shall replace the rectangle $R$ by an infinite strip of the form $\mathbf{R}_{x} \times[0, \pi]_{y}$ and consider the wave evolution of the functions $e_{k}(x, y)=\varphi(x) \sin k y$, $\varphi \in S(\mathbf{R}), \widehat{\varphi} \in C_{0}^{\infty}(\mathbf{R}),\|\varphi\|_{L^{2}}=1$. Here $\widehat{\varphi}(\xi)=\int e^{-i x \xi} \varphi(x) d x$ is the Fourier transform of $\varphi$. Then $e_{k}$ is an $O(1)$-quasimode for the Dirichlet Laplacian $\Delta_{D}$ in $\mathbf{R}_{x} \times[0, \pi]_{y}$, and a simple calculation shows that

$$
\left(\cos t \sqrt{-\Delta_{D}}\right) e_{k}=(\cos t k) e_{k}+O\left(\frac{t}{k}\right), \quad|t| \leq k \rightarrow \infty .
$$

These heuristic considerations suggest that the optimal energy decay estimate in Theorem 1.1, modulo an $\varepsilon$-loss, should be of the form

$$
E(u, t)^{1 / 2} \leq \frac{O_{\varepsilon}(1)}{t^{1-\varepsilon}}\left\|\left(u_{0}, u_{1}\right)\right\|_{D(A)}, \quad \varepsilon>0 .
$$

(Remark that taking $t=\varepsilon k, \varepsilon \ll 1$ in (1.6) and letting $k$ tend to infinity shows that (1.7) cannot hold if $\varepsilon<0$.)

In section 3 we shall describe a class of examples of $C^{\infty}$-damping coefficients $a$ for which we are able to improve the result of Theorem 1.1 and obtain an estimate of the form (1.7).

Theorem 1.2. Assume that $a \in C^{\infty}(\bar{\Omega})$ is a damping coefficient satisfying the assumptions of the beginning of section 3 and in particular (3.1), (3.2), for some $m>4$. Then we have for $k>0$ and $\varepsilon>0$,

$$
E(u, t)^{1 / 2} \leq \frac{O_{k, \varepsilon}(1)}{t^{\frac{k}{1+\frac{4}{m}}}-\varepsilon}\left\|\left(u_{0}, u_{1}\right)\right\|_{D\left(A^{k}\right)}, \quad t \geq 2 .
$$

In particular if (3.2) is satisfied for all $m>4$, then for each $k>0$ and each $\varepsilon>0$ there exists $C_{k, \varepsilon}>0$ such that for each $\left(u_{0}, u_{1}\right) \in D\left(A^{k}\right)$ we have

$$
E(u, t)^{1 / 2} \leq \frac{C_{k, \varepsilon}}{t^{k-\varepsilon}}\left\|\left(u_{0}, u_{1}\right)\right\|_{D\left(A^{k}\right)}, \quad t \geq 2 .
$$

At the present time, we do not know whether one can obtain an estimate of the form (1.7) for a general continuous, or even smooth, damping term, concentrated near the non-rectangular part of the domain.

Remark. When this paper was completed, the authors learned of a very recent work by Kim Dang Phung [9], where a polynomial decay estimate for the energy, with an unspecified decay rate, was obtained on a partially cubic domain, with the damping acting in a neighborhood of the boundary except between a pair of parallel square faces of the cube. An initial glance at [9] shows that the methods of the present paper are completely different. 
Acknowledgment. The second author is grateful to the National Science Foundation for partial support under the grant DMS-0304970. He would also like to thank Rowan Killip for a stimulating discussion.

\section{Proof of Theorem 1.1}

Following [4] and [8], we shall use the stationary methods, and the main point will be to estimate the resolvent $(\lambda-A)^{-1}: H \rightarrow H$ for $\lambda \in \mathbf{R},|\lambda| \gg 1$.

The following result, closely related to Proposition 6.1 of [4], is our starting point.

Proposition 2.1. Let $R=[0,1]_{x} \times[0, \pi]_{y} \subset \mathbf{R}^{2}$ be a rectangle, and let us consider the stationary problem

$$
\left(-\Delta+2 i a \lambda-\lambda^{2}\right) u=f+\partial_{x} g,\left.\quad u\right|_{\partial R}=0 .
$$

Here $\lambda \in \mathbf{R},|\lambda| \geq 1$ and $f, g \in L^{2}(R)$. Assume that $0 \leq a \in C(R)$ is such that

$$
[0,1] \ni x \mapsto \inf _{y \in[0, \pi]} a(x, y) \text { does not vanish identically. }
$$

Then we have

$$
\|u\|_{L^{2}(R)}^{2} \leq O(1)\left(\|f\|_{L^{2}(R)}^{2}+\|g\|_{L^{2}(R)}^{2}+\lambda^{2} \int_{R} a(x, y)|u|^{2} d x d y\right) .
$$

Proof. When establishing (2.3), it suffices to do so when $a=a(x)$ is a function of $x$ only. Indeed, assume that (2.3) has already been established in this special case. If $a \in C(R)$ satisfies $(2.2)$, we can take $0 \leq a_{1} \in C([0,1])$, not identically zero and such that $0 \leq a_{1}(x) \leq a(x, y)$ when $(x, y) \in R$. Now if $u \in H_{0}^{1}(R)$ satisfies

$$
\left(-\Delta+2 i \lambda a-\lambda^{2}\right) u=f+\partial_{x} g,
$$

then

$$
\left(-\Delta+2 i \lambda a_{1}-\lambda^{2}\right) u=f+\partial_{x} g+2 i \lambda\left(a_{1}-a\right) u .
$$

Applying (2.3) to (2.5), we get

$$
\|u\|_{L^{2}}^{2} \leq O(1)\left(\|f\|_{L^{2}}^{2}+\|g\|_{L^{2}}^{2}+\lambda^{2} \int_{R}\left(a-a_{1}\right)|u|^{2} d x d y+\lambda^{2} \int_{R} a_{1}|u|^{2} d x d y\right),
$$

and the bound (2.3) follows in the general case. In what follows, when proving (2.3), we shall therefore assume that $0 \leq a=a(x) \in C([0,1])$ is a function of $x$ only, which does not vanish identically.

When analyzing (2.1), we follow [4], [5] and separate variables. The Dirichlet realization of $-\partial_{y}^{2}$ on $L^{2}((0, \pi))$ has the eigenvalues $k^{2}, k=1,2, \ldots$ with the corresponding eigenfunctions $e_{k}(y)=\sqrt{2 / \pi} \sin k y$, forming an orthonormal basis in $L^{2}((0, \pi))$. Writing

$$
u(x, y)=\sum_{k=1}^{\infty} u_{k}(x) e_{k}(y)
$$

and similarly for $f$ and $g$,

$$
f(x, y)=\sum_{k=1}^{\infty} f_{k}(x) e_{k}(y), \quad g(x, y)=\sum_{k=1}^{\infty} g_{k}(x) e_{k}(y)
$$


we see that $u_{k}, k=1,2, \ldots$ satisfy

$$
\left(-\partial_{x}^{2}+2 i a(x) \lambda+k^{2}-\lambda^{2}\right) u_{k}(x)=f_{k}(x)+\partial_{x} g_{k}(x), \quad u_{k}(0)=u_{k}(1)=0 .
$$

When analyzing (2.7), we shall first consider the case when $k \in \mathbf{N}$ is such that $k \geq|\lambda|$. We then claim that

$$
\left\|u_{k}\right\|_{L^{2}}^{2} \leq O(1)\left(\left\|f_{k}\right\|_{L^{2}}^{2}+\left\|g_{k}\right\|_{L^{2}}^{2}\right),
$$

and here we do not need the assumption that $a$ does not vanish identically.

Indeed, multiplying the first equation in $(2.7)$ by $\overline{u_{k}}$, we obtain, integrating by parts and taking the real part, that

$$
\begin{aligned}
& \int_{0}^{1}\left|u_{k}^{\prime}(x)\right|^{2} d x+\left(k^{2}-\lambda^{2}\right) \int_{0}^{1}\left|u_{k}(x)\right|^{2} d x \\
& =\operatorname{Re} \int_{0}^{1}\left(f_{k}(x) \overline{u_{k}}(x)-g_{k}(x) \partial_{x} \overline{u_{k}}(x)\right) d x .
\end{aligned}
$$

Here the right hand side does not exceed

$$
\left(\left\|f_{k}\right\|_{L^{2}}+\left\|g_{k}\right\|_{L^{2}}\right)\left(\left\|u_{k}\right\|_{L^{2}}+\left\|u_{k}^{\prime}\right\|_{L^{2}}\right) \leq 2\left(\left\|f_{k}\right\|_{L^{2}}+\left\|g_{k}\right\|_{L^{2}}\right)\left\|u_{k}^{\prime}\right\|_{L^{2}},
$$

where we also used the Poincaré inequality

$$
\left\|u_{k}\right\|_{L^{2}} \leq\left\|u_{k}^{\prime}\right\|_{L^{2}}, \quad u_{k} \in H_{0}^{1}((0,1)) .
$$

Another application of the Poincaré inequality combined with (2.9) then immediately gives (2.8).

We now come to discuss the second case when $k<|\lambda|$. Let $\tau \in \mathbf{R}$ be such that $\tau^{2}=\lambda^{2}-k^{2}$. Then we have

$$
\begin{aligned}
\left(-\partial_{x}^{2}+2 i a(x) \tau-\tau^{2}\right) u_{k}(x) & =f_{k}(x)+\partial_{x} g_{k}(x)+2 i(\tau-\lambda) a(x) u_{k}(x), \\
u_{k}(0) & =u_{k}(1)=0 .
\end{aligned}
$$

Now the expression in the left hand side of (2.10) is a one-dimensional stationary damped wave operator, for which it is well-known [6] that the resolvent bound

$$
R_{0}(\tau):=\left(-\partial_{x}^{2}+2 i a \tau-\tau^{2}\right)^{-1}=O\left(\frac{1}{1+|\tau|}\right): L^{2}((0,1)) \rightarrow L^{2}((0,1))
$$

holds true, since $0 \leq a \in C([0,1])$ is not identically zero. See also [7]. It follows that

$$
R_{0}(\tau)=O(1): L^{2}((0,1)) \rightarrow H_{0}^{1}((0,1)), \quad \tau \in \mathbf{R},
$$

and hence by duality, the same bound holds when $R_{0}(\tau)$ is viewed as an operator from $H^{-1}((0,1))$ to $L^{2}((0,1))$. Applying these observations to $(2.10)$, we get

$$
\left\|u_{k}\right\|_{L^{2}}^{2} \leq O(1)\left(\left\|f_{k}\right\|_{L^{2}}^{2}+\left\|g_{k}\right\|_{L^{2}}^{2}\right)+O(1) \lambda^{2}\left\|a^{1 / 2} u_{k}\right\|_{L^{2}}^{2} .
$$

Combining the bounds (2.8) and (2.13) and summing with respect to $k$, we get the proposition.

Proposition 2.2. Let $\Omega \subset \mathbf{R}^{n}$ be a partially rectangular domain, $\Omega=R \cup W$, and let $0 \leq a \in C(\bar{\Omega})$ be such that $a>0$ in $\bar{W}$. If we put $R(\lambda)=\left(-\Delta+2 i a \lambda-\lambda^{2}\right)^{-1}$, $\lambda \in \mathbf{R}$, then we have

$$
R(\lambda)=O(|\lambda|): L^{2}(\Omega) \rightarrow L^{2}(\Omega), \quad|\lambda| \geq 1 .
$$


Proof. We may assume that $R=[0,1]_{x} \times[0, \pi]_{y}$, with the sides parallel to the $x$-axis contained in the boundary of $\Omega$.

When $f \in L^{2}(\Omega)$, let $u \in H_{0}^{1}(\Omega)$ be the solution of

$$
\left(-\Delta+2 i a \lambda-\lambda^{2}\right) u=f,\left.\quad u\right|_{\partial \Omega}=0, \quad|\lambda| \geq 1 .
$$

We now let $0 \leq \chi \in C_{0}^{\infty}((0,1)), 0 \leq \chi \leq 1$, be a cut-off function such that $\chi=1$ on $[\epsilon, 1-\epsilon]$. Here $\epsilon>0$ small is to be chosen. Then it follows that $\chi u$, viewed as a function on $R$, vanishes on its boundary and satisfies in the interior

$$
\left(-\Delta+2 i a \lambda-\lambda^{2}\right) \chi u=\chi f+[-\Delta, \chi] u .
$$

Here $[-\Delta, \chi] u=\chi^{\prime \prime}(x) u-2 \partial_{x}\left(\chi^{\prime}(x) u\right)$. An application of Proposition 2.1 gives therefore that

$$
\|\chi u\|_{L^{2}}^{2} \leq O(1)\left(\|f\|_{L^{2}}^{2}+\int_{\omega_{\epsilon}}|u|^{2} d x d y+|\lambda|^{2} \int_{R} a(x, y)|u|^{2} d x d y\right) .
$$

Here $\omega_{\epsilon}$ is a neighborhood of the support of $\chi^{\prime}(x)$, and choosing $\epsilon>0$ small enough, we can achieve that $\omega_{\epsilon}$ is so close to the vertical sides of $\partial R$, that it is contained in the set where $a(x, y)$ is bounded away from 0 . It follows that

$$
\|\chi u\|_{L^{2}}^{2} \leq O(1)\|f\|_{L^{2}}^{2}+O(1)|\lambda|^{2} \int_{\Omega} a(x, y)|u(x, y)|^{2} d x d y .
$$

Now observe that multiplying (2.15) by $\bar{u}$, we obtain, integrating by parts and taking the imaginary part, that

$$
2 \lambda \int_{\Omega} a|u|^{2} d x d y=\operatorname{Im} \int_{\Omega} f \bar{u} d x d y .
$$

Hence

$$
|\lambda| \int_{\Omega} a|u|^{2} d x d y \leq\|f\|_{L^{2}}\|u\|_{L^{2}} .
$$

and using this in (2.18) we see that

$$
\|\chi u\|_{L^{2}}^{2} \leq O(1)\|f\|_{L^{2}}^{2}+O(1)|\lambda|\|f\|_{L^{2}}\|u\|_{L^{2}} .
$$

It remains to estimate the $L^{2}$-norm of $(1-\chi) u$, and to that end we notice that the support of this function is contained in the set where $a$ is bounded away from zero. Therefore, another application of (2.20) shows that

$$
\|(1-\chi) u\|_{L^{2}}^{2} \leq O(1)\|f\|_{L^{2}}\|u\|_{L^{2}}, \quad|\lambda| \geq 1 .
$$

Putting together the estimates (2.21) and (2.22) we get

$$
\|u\|_{L^{2}} \leq O(1)\|f\|_{L^{2}}+O(1)|\lambda|^{1 / 2}\|f\|_{L^{2}}^{1 / 2}\|u\|_{L^{2}}^{1 / 2},
$$

so that

$$
\|u\|_{L^{2}} \leq O(1)|\lambda|\|f\|_{L^{2}}
$$

This completes the proof. 
With Proposition 2.2 available, we are in the position to estimate the norm of the resolvent of the operator $A$ in (1.2) on the real axis. In doing so, we notice that a simple computation, as in [8] and [7], shows that

$$
(\lambda-A)^{-1}=\left(\begin{array}{cc}
R(\lambda)(2 i a-\lambda) & -R(\lambda) \\
R(\lambda)\left(2 i a \lambda-\lambda^{2}\right)-1 & -\lambda R(\lambda)
\end{array}\right), \quad \lambda \in \mathbf{R} .
$$

Here, as in Proposition 2.2, we have written $R(\lambda)=\left(-\Delta+2 i \lambda a-\lambda^{2}\right)^{-1}$. To estimate the norm $(\lambda-A)^{-1}$ as an operator on $H=H_{0}^{1} \times L^{2}$, we have to derive bounds on the operators

$$
R(\lambda): L^{2} \rightarrow H_{0}^{1}, \quad R(\lambda)\left(2 i a \lambda-\lambda^{2}\right)-1: H_{0}^{1} \rightarrow L^{2},
$$

and

$$
R(\lambda)(2 i a-\lambda): H_{0}^{1} \rightarrow H_{0}^{1} .
$$

Now Proposition 2.2 together with an integration by parts argument shows that

$$
R(\lambda)=O\left(\lambda^{2}\right): L^{2} \rightarrow H_{0}^{1},
$$

and hence by duality,

$$
R(\lambda)=O\left(\lambda^{2}\right): H^{-1} \rightarrow L^{2} .
$$

When estimating the norm of the second operator in (2.26), we use that

$$
R(\lambda)\left(2 i a \lambda-\lambda^{2}\right)-1=R(\lambda) \Delta,
$$

and hence, combining (2.29) together with the fact that the Laplacian $\Delta: H_{0}^{1} \rightarrow H^{-1}$ is continuous, we get

$$
R(\lambda)\left(2 i a \lambda-\lambda^{2}\right)-1=R(\lambda) \Delta=O\left(\lambda^{2}\right): H_{0}^{1} \rightarrow L^{2} .
$$

It remains to estimate the norm in (2.27), and to that end, we write

$$
R(\lambda)(2 i a-\lambda)=\frac{1}{\lambda}(1+R(\lambda) \Delta) .
$$

If $f \in H_{0}^{1}$ and $u=R(\lambda) \Delta f \in H_{0}^{1}$ then

$$
\left(-\Delta+2 i a \lambda-\lambda^{2}\right) u=\Delta f \in H^{-1},
$$

and hence multiplying (2.32) by $\bar{u}$, integrating by parts, and taking the real part, we get

$$
\|u\|_{H_{0}^{1}}^{2}-\lambda^{2}\|u\|_{L^{2}}^{2} \leq\|\Delta f\|_{H^{-1}}\|u\|_{H_{0}^{1}} \leq\|f\|_{H_{0}^{1}}\|u\|_{H_{0}^{1}} .
$$

Therefore,

$$
\|u\|_{H_{0}^{1}}^{2} \leq O(1)\left(\lambda^{2}\|u\|_{L^{2}}^{2}+\|f\|_{H_{0}^{1}}^{2}\right) .
$$

When estimating the $L^{2}$-norm of $u=R(\lambda) \Delta f$, we use (2.30) to conclude that

$$
\|u\|_{L^{2}} \leq O\left(\lambda^{2}\right)\|f\|_{H_{0}^{1}}
$$

and combining this estimate with (2.34) we get

$$
\|u\|_{H_{0}^{1}} \leq O\left(|\lambda|^{3}\right)\|f\|_{H_{0}^{1}} .
$$

An application of (2.31) then shows that

$$
R(\lambda)(2 i a-\lambda)=O\left(\lambda^{2}\right): H_{0}^{1} \rightarrow H_{0}^{1} .
$$


Combining Proposition 2.2 together with (2.28), (2.30), (2.37), and the fact that $A$ has no real eigenvalues, we get the basic bound

$$
(\lambda-A)^{-1}=O\left((1+|\lambda|)^{2}\right): H \rightarrow H, \lambda \in \mathbf{R} .
$$

We shall finally show how the bound (2.38) allows us to conclude the proof of Theorem 1.1. In doing so, we shall follow the argument of [8] closely, adapting it to the present case.

When $k>1$ is an integer and $x \in H$, we write, as in [8] and [3], for $t>0$,

$$
e^{i t A}(1-i A)^{-k} x=\frac{1}{2 \pi i} \int_{\gamma} e^{i t \lambda} \frac{1}{(1-i \lambda)^{k}}(\lambda-A)^{-1} x d \lambda .
$$

Here $\gamma=\{\lambda \in \mathbf{C} ; \lambda=\eta-i / 2, \eta \in \mathbf{R}\}$. Furthermore, when

$$
X=X(t)=\gamma_{1}\left(\frac{t}{\log t}\right)^{1 / 2}
$$

where $\gamma_{1}>0$ is to be chosen, we use the same decomposition of (2.39) as in [8],

$$
\begin{aligned}
& e^{i t A}(1-i A)^{-k} x \\
= & \frac{1}{2 \pi i} \frac{1}{\sqrt{2 \pi}} \int_{\gamma} \int_{\mathbf{R}} e^{i t \lambda} \frac{1}{(1-i \lambda)^{k}} e^{-(\lambda-\tau)^{2} / 2}(\lambda-A)^{-1} x d \tau d \lambda \\
= & \int_{\gamma} \int_{|\tau| \leq X} \cdots+\int_{\gamma} \int_{|\tau| \geq X} \cdots=I_{1}+I_{2},
\end{aligned}
$$

where

$$
I_{1}=\frac{1}{2 \pi i} \frac{1}{\sqrt{2 \pi}} \int_{\gamma} \int_{|\tau| \leq X} e^{i t \lambda} \frac{1}{(1-i \lambda)^{k}} e^{-(\tau-\lambda)^{2} / 2}(\lambda-A)^{-1} x d \tau d \lambda
$$

and

$$
I_{2}=\frac{1}{2 \pi i} \frac{1}{\sqrt{2 \pi}} \int_{\gamma} \int_{|\tau| \geq X} e^{i t \lambda} \frac{1}{(1-i \lambda)^{k}} e^{-(\tau-\lambda)^{2} / 2}(\lambda-A)^{-1} x d \tau d \lambda
$$

Now combining (2.38) together with a perturbation argument, we see that the function $\lambda \mapsto(\lambda-A)^{-1} x$ is holomorphic in $\lambda$ with values in $H$, in the region below and including the curve

$$
\gamma_{\epsilon_{0}}=\left\{\lambda=\eta+i \frac{\epsilon_{0}}{\eta^{2}},|\eta| \geq 1\right\} \cup\left\{\lambda=\eta+i \epsilon_{0},|\eta| \leq 1\right\},
$$

where $\epsilon_{0}>0$ is small enough but fixed. Also, we see that along $\gamma_{\epsilon_{0}},(\lambda-A)^{-1} x$ is bounded in $H$ by $O(1)(1+|\eta|)^{2}\|x\|_{H}$. Therefore, when estimating $I_{1}$, we may write

$$
I_{1}=\frac{1}{2 \pi i} \frac{1}{\sqrt{2 \pi}} \int_{\gamma_{\epsilon_{0}}} \int_{|\tau| \leq X} e^{i t \lambda} \frac{1}{(1-i \lambda)^{k}} e^{-(\tau-\lambda)^{2} / 2}(\lambda-A)^{-1} x d \tau d \lambda .
$$

The contribution to $I_{1}$ coming from the part of $\gamma_{\epsilon_{0}}$ where $|\eta| \leq 1$ is easily seen to be bounded by $O\left(e^{-\epsilon_{0} t} X(t)\right)\|x\|_{H}$, hence decaying exponentially, and therefore we may concentrate on the part of $\gamma_{\epsilon_{0}}$ where $|\eta| \geq 1$. When estimating the contribution coming from this part, exactly as in [8], we treat separately the cases when $1 \leq|\eta| \leq$ 
$\gamma_{2}(t / \log t)^{1 / 2}$ and $|\eta| \geq \gamma_{2}(t / \log t)^{1 / 2}$. Here $\gamma_{2}>0$ is to be chosen. The $H$-norm of the integrand in $I_{1}$ coming the part where $|\eta| \geq 1$ is bounded by

$$
O(1) \exp \left(-\frac{t \epsilon_{0}}{|\eta|^{2}}\right) \frac{|\eta|^{2}}{(1+|\eta|)^{k}} e^{-(\tau-\eta)^{2} / 2}\|x\|_{H} .
$$

Here $|\tau| \leq \gamma_{1}(t / \log t)^{1 / 2}$. It follows that the contribution to $I_{1}$ coming from the region where $1 \leq|\eta| \leq \gamma_{2}(t / \log t)^{1 / 2}$ is controlled by

$$
O(1) \frac{t}{\log t} \frac{1}{t^{\epsilon_{0} / \gamma_{2}^{2}}}\|x\|_{H}
$$

which decays as any fixed inverse power of $t$, provided that $\gamma_{2}>0$ is sufficiently small. Having fixed $\gamma_{2}>0$ small enough, as in [8], we choose $\gamma_{1} \in\left(0, \gamma_{2}\right)$, and observe that for $|\tau| \leq \gamma_{1}(t / \log t)^{1 / 2}$ and $|\eta| \geq \gamma_{2}(t / \log t)^{1 / 2}$, we have

$$
\frac{1}{2}(\tau-\eta)^{2} \geq \delta\left(\tau^{2}+\eta^{2}\right), \quad \delta>0 .
$$

It follows then that the final contribution to $I_{1}$ coming from the region where $|\eta| \geq$ $\gamma_{2}(t / \log t)^{1 / 2}$ is obtained by integrating over this region

$$
O(1)\left(\frac{t}{\log t}\right)^{1 / 2} \frac{|\eta|^{2}}{(1+|\eta|)^{k}} e^{-\delta \eta^{2}}\|x\|_{H}
$$

and this clearly decays rapidly (and even exponentially) as $t \rightarrow \infty$. We conclude that for each $N \in \mathbf{N}$,

$$
I_{1}=O_{N}\left(t^{-N}\right)\|x\|_{H} .
$$

When estimating $I_{2}$ in (2.42), we continue to follow [8] and write

$$
I_{2}=e^{i t A} J
$$

where

$$
J=\frac{1}{2 \pi i} \frac{1}{\sqrt{2 \pi}} \int_{|\tau| \geq X} \int_{\gamma} \frac{1}{(1-i \lambda)^{k}} e^{-(\tau-\lambda)^{2} / 2}(\lambda-A)^{-1} x d \tau d \lambda .
$$

Since $e^{i t A}$ is uniformly bounded on $H$ for $t \geq 0$, it suffices to estimate $J$ in the energy norm, as $t \rightarrow \infty$. Exactly as in [8], we then see that we have to estimate the integral

$$
\int_{0}^{\infty} S_{X-\frac{i}{2}+\mu e^{-i \pi / 8}}(x) d \mu
$$

where

$$
S_{\tau}(x)=\int_{\gamma} \frac{1}{(1-i \lambda)^{k}} e^{-(\lambda-\tau)^{2}}(\lambda-A)^{-1} x d \lambda .
$$

Arguing precisely as in [8] and making a contour deformation as in that paper, we then verify that with $\tau=X-\frac{i}{2}+\mu e^{-i \pi / 8}, \mu \geq 0$,

$$
\left\|S_{\tau}(x)\right\|_{H} \leq O(1)\left(e^{-\delta\left(\mu^{2}+X^{2}\right)}+\frac{1}{(1+\mu)(\mu+X)^{k}}\right)\|x\|_{H}, \quad \delta>0 .
$$

Let us remark here that when deriving (2.54), the bound (1.3) is important. 
It follows from (2.50), (2.52), and (2.54) that the $H$-norm of $I_{2}$ does not exceed a constant times the norm of the vector $x$ multiplied by

$$
\int_{0}^{\infty}\left(e^{\left(-\delta\left(\mu^{2}+X^{2}\right)\right)}+\frac{1}{(1+\mu)(\mu+X)^{k}}\right) d \mu
$$

Since

$$
\int_{0}^{\infty} \frac{d \mu}{(\mu+1)(\mu+X)^{k}}=O_{k}\left(\frac{\log X}{X^{k}}\right),
$$

we conclude, recalling the definition of $X$, that

$$
I_{2}=O_{k}(1) \frac{(\log t)^{k / 2+1}}{t^{k / 2}}\|x\|_{H}, \quad t \geq 2, \quad k=2,3, \ldots
$$

This completes the proof of Theorem 1.1 in the case when $k>1$ is an integer. Using an interpolation argument as explained in [3], we get the result for a general $k>0$.

Remark. By refining again the analysis above as in section 4 of [3] one could probably avoid the logarithmic loss in (2.57).

\section{Improved decay estimates: an example}

In this section, we shall give an example of a class of damping coefficients vanishing away from a neighborhood of the non-rectangular part of the domain, for which the result of Theorem 1.1 can be improved, leading to an estimate of the type (1.7). When doing so, to fix the ideas, we shall let $\Omega=S$ be the Bunimovich stadium, defined in the example preceding the formulation of Theorem 1.1.

When $S=R \cup W, R=[0,1]_{x} \times[0, \pi]_{y}$, we let $0 \leq a \in C^{\infty}(\bar{S})$ be such that $a>0$ in $\bar{W}$. We assume that

$$
a^{-1}(0)=[\delta,(1-\delta)]_{x} \times[0, \pi]_{y}, \quad 0<\delta \ll 1,
$$

and that $a$ is independent of $y$ when $x$ is close to $\delta$ and $1-\delta$. Furthermore, let us assume for simplicity that close to $\delta$ (respectively $1-\delta$ ), we have

$$
\left.\frac{d^{m}}{d x^{m}} a(x) \leq 0 \quad \text { (respectively } \geq 0\right),
$$

for some $m \geq 4$. We then immediately deduce the following result.

Lemma 3.1. For any $n<m$ there exists $C_{n, m}>0$ such that

$$
\left|a^{(n)}(x)\right| \leq C_{n, m} a(x)^{\frac{m-n}{m}} .
$$

Proof. It suffices to consider the case $x \geq(1-\delta)$. Using Taylor's formula, we get for $x \geq(1-\delta)$,

$$
a(x)=\int_{1-\delta}^{x} \frac{(x-s)^{m-1}}{(m-1) !} a^{(m)}(s) d s, \quad a^{\prime}(x)=\int_{1-\delta}^{x} \frac{(x-s)^{m-2}}{(m-2) !} a^{(m)}(s) d s .
$$

As a consequence,

$$
\left|a^{\prime}(x)\right| \leq \int_{(1-\delta)}^{y} \frac{(x-s)^{m-2}}{(m-2) !} a^{(m)}(s) d s+\int_{y}^{x} \frac{(x-s)^{m-2}}{(m-2) !} a^{(m)}(s) d s,
$$


with $(1-\delta)<y<x$ to be chosen. To estimate the first integral in (3.3) we use the bound

$$
\frac{(x-s)^{m-2}}{(m-2) !}=\frac{(m-1)}{x-s} \frac{(x-s)^{m-1}}{(m-1) !} \leq \frac{(m-1)}{x-y} \frac{(x-s)^{m-1}}{(m-1 !}
$$

and for the second integral, we use that

$$
\frac{(x-s)^{m-2}}{(m-2) !} \leq \frac{(x-y)^{m-2}}{(m-2) !} .
$$

We obtain

$$
\left|a^{\prime}(x)\right| \leq \frac{(m-1)}{(x-y)} a(x)+O_{m}(1)(x-y)^{m-1} .
$$

Choosing $y$ so that $a(x)=(x-y)^{m}$ gives the lemma for $n=1$. The general case is similar.

Let us consider now the stationary problem

$$
\left(-\Delta+2 i \lambda a-\lambda^{2}\right) u=f \in L^{2}(S),\left.\quad u\right|_{\partial S}=0, \quad \lambda \gg 1 .
$$

As in the proof of Proposition 2.2, we let $0 \leq \chi_{\lambda} \in C_{0}^{\infty}((0,1))$ be a cut-off function so that $\chi_{\lambda}$ vanishes for $x$ close to 0 or 1 .Then the function $\chi_{\lambda} u$ vanishes on $\partial R$ and satisfies in the interior of the rectangle,

$$
\left(-\Delta-\lambda^{2}\right) \chi_{\lambda} u=\chi f+\chi_{\lambda}^{\prime \prime} u-2 \partial_{x}\left(\chi_{\lambda}^{\prime} u\right)-2 i \lambda a(x) \chi_{\lambda} u .
$$

We choose $\chi_{\lambda}=\chi(\lambda a(x))$ with $\chi=0$ for $|x| \geq 2$, and $\chi=1$ for $|x| \leq 1$, so that in the support of $\chi_{\lambda}^{\prime}(x)$ we have

$$
a(x) \sim \frac{1}{\lambda}, \quad \lambda \gg 1 .
$$

From Lemma 3.1 we get the following bounds on the derivative of $\chi_{\lambda}$,

$$
\left|\chi_{\lambda}^{\prime}\right|=\left|\lambda \chi^{\prime}(\lambda a(x)) a^{\prime}(x)\right| \leq O(1) \lambda^{\frac{1}{m}},
$$

and similarly

$$
\left|\chi_{\lambda}^{(n)}\right| \leq O(1) \lambda^{\frac{n}{m}}, \quad n<m .
$$

We now come to estimate the $L^{2}$-norms of the functions $\chi_{\lambda}^{\prime \prime} u, \chi_{\lambda}^{\prime} u$, and $\lambda a(x) \chi_{\lambda} u$, occurring in the right hand side of (3.5). In doing so, we write

$$
\chi_{\lambda}^{\prime} u=\frac{\chi_{\lambda}^{\prime} a^{1 / 2} u}{a^{1 / 2}}
$$

and using (3.6), (3.7), we get

$$
\left|\frac{\chi_{\lambda}^{\prime}}{a^{1 / 2}}\right| \leq O(1) \lambda^{\frac{1}{2}+\frac{1}{m}}
$$

and consequently

$$
\left\|\chi_{\lambda}^{\prime} u\right\|_{L^{2}(R)} \leq O(1) \lambda^{\frac{1}{2}+\frac{1}{m}}\left\|a^{1 / 2} u\right\|_{L^{2}(S)} .
$$

Estimating the $L^{2}$-norm of $\chi_{\lambda}^{\prime \prime} u$ in a similar way, we get

$$
\left\|\chi_{\lambda}^{\prime \prime} u\right\|_{L^{2}} \leq O(1) \lambda^{\frac{1}{2}+\frac{2}{m}}\left\|a^{1 / 2} u\right\|_{L^{2}(S)} .
$$


Finally, a similar argument shows that

$$
\left\|a \lambda \chi_{\lambda} u\right\|_{L^{2}(R)} \leq O(1) \lambda^{1 / 2}\left\|a^{1 / 2} u\right\|_{L^{2}(S)} .
$$

At this point we can apply Proposition 6.1 of [4] to (3.5) directly, choosing $\omega_{x}$ there to be a neighborhood of the edges $x=0$ and $x=1$ where $a$ is bounded from below and consequently $\chi_{\lambda} u$ vanishes. Using (3.9), (3.10), and (3.11), we get, with $\omega=\omega_{x} \times[0, \pi]_{y}$,

$$
\left\|\chi_{\lambda} u\right\|_{L^{2}(R)}^{2} \leq O(1)\left(\|f\|_{L^{2}(R)}^{2}+\lambda^{1+\frac{4}{m}}\left\|a^{1 / 2} u\right\|_{L^{2}(S)}^{2}\right) .
$$

An application of (2.20) in (3.12) gives next

$$
\left\|\chi_{\lambda} u\right\|_{L^{2}}^{2} \leq O(1)\left(\|f\|_{L^{2}}^{2}+\lambda^{\frac{4}{m}}\|f\|_{L^{2}}\|u\|_{L^{2}}\right) .
$$

It remains to control the $L^{2}$-norm of $\left(1-\chi_{\lambda}\right) u$, and when doing so we remark that the support of $1-\chi_{\lambda}$ is contained in the set where $a \geq 1 / \lambda, \lambda \gg 1$. Using (2.20) for the second time, we infer that

$$
\left\|\left(1-\chi_{\lambda}\right) u\right\|_{L^{2}}^{2} \leq O(1)\|f\|_{L^{2}}\|u\|_{L^{2}} .
$$

Putting together (3.13) and (3.14) we get the estimate

$$
\|u\|_{L^{2}} \leq O(1)\left(\|f\|_{L^{2}}+\lambda^{\frac{2}{m}}\|f\|_{L^{2}}^{1 / 2}\|u\|_{L^{2}}^{1 / 2}\right)
$$

and finally,

$$
\|u\|_{L^{2}} \leq O(1)|\lambda|^{\frac{4}{m}}\|f\|_{L^{2}}, \quad \lambda \in \mathbf{R},|\lambda| \gg 1 .
$$

Repeating the arguments of section 2, with the bound (3.16) in place of Proposition 2.2 , we get the bound

$$
(\lambda-A)^{-1}=O(1)(1+|\lambda|)^{1+\frac{4}{m}}: H \rightarrow H, \quad \lambda \in \mathbf{R},
$$

and Theorem 1.2 follows as before.

\section{References}

[1] C. Bardos, G. Lebeau, and J. Rauch, Sharp sufficient conditions for the observation, control, and stabilization of waves from the boundary, SIAM J. Control Optim. 30 (1992), no. 5, 10241065.

[2] L. Bunimovich, On the ergodic properties of nowhere dispersing billiards, Comm. Math. Phys. 65 (1979), no. 3, 295-312.

[3] N. Burq, Décroissance de l'énergie locale de l'équation des ondes pour le problème extérieur et absence de résonance au voisinage du réel, Acta Math. 180 (1998), no. 1, 1-29.

[4] N. Burq and M. Zworski, Geometric control in the presence of a black box, J. Amer. Math. Soc. 17 (2004), no. 2, 443-471.

[5] _ Bouncing ball modes and quantum chaos, SIAM Rev. 47 (2005), no. 1, 43-49.

[6] S. Cox and E. Zuazua, The rate at which the energy decays in a damped string, Comm. Partial Differential Equations 19 (1994), no. 1-2, 213-243.

[7] M. Hitrik, Eigenfrequencies and expansions for damped wave equations, Methods Appl. Anal. 10 (2003), no. 4, 543-564.

[8] G. Lebeau, Équation des ondes amorties. Algebraic and geometric methods in mathematical physics (Kaciveli 1993), 73-109, Math. Phys. Stud. 19, Kluwer Acad. Publ., Dordrecht, 1996.

[9] K. D. Phung, Polynomial decay rate for the dissipative wave equation, Preprint 2005. www.arxiv.org/abs/math.AP/0312281. 
Département de Mathématiques, Université Paris Sud-Orsay, 91405 Orsay Cedex, France, and Institut Universitaire DE France

E-mail address: nicolas.burq@math.u-psud.fr

Department of Mathematics, University of California, Los Angeles, CA 90095

E-mail address: hitrik@math.ucla.edu 